\title{
Practicable Measures and Indices of Insulin Resistance in Nutrition Research
}

\author{
John G. Hattersley • Martin O. Weickert
}

Published online: 28 August 2013

(C) Springer Science+Business Media New York 2013

\begin{abstract}
Insulin resistance (IR) is an early onset key factor in the development of type 2 diabetes, and can be improved by lifestyle measures including weight loss, physical activity, and modulation of the macronutrient composition of the diet. An accurate measurement for IR is essential when investigating effects of lifestyle in dietary interventions and epidemiological research. Whilst sensitivity and specificity are of prime importance, practicalities of resource requirements, equipment, and expertise in nutrition research units need to be considered. There are direct methods of measuring IR, referred collectively as clamp and stable isotope or tracer techniques; however, these fail to meet requirements of practical measurements. Therefore, surrogate markers have been developed. In this article the most commonly used methods will be reviewed, in terms of feasibility to research studies, particularly nutritional studies, which due to relatively small perturbation offer a unique set of problems for researchers.
\end{abstract}

Keywords Euglycemic hyperinsulinemic clamp $\cdot$ Stable isotope clamps $\cdot$ Surrogate indices $\cdot$ Nutritional research . Dietary intervention · HOMA · Minimal model · OGIS . Hepatic insulin resistance $\cdot$ OGTT $\cdot$ FSIVGTT

John G. Hattersley and Martin O. Weickert contributed equally to this work

J. G. Hattersley

Research and Development, University Hospitals Coventry and Warwickshire NHS Trust, Coventry, UK

J. G. Hattersley

School of Engineering, University of Warwick, Coventry, UK

\section{O. Weickert $(\square)$}

Warwickshire Institute for the Study of Diabetes, Endocrinology and Metabolism, University Hospitals Coventry and Warwickshire NHS Trust, Clifford Bridge Road, CV2 2DX Coventry, UK

e-mail: m.weickert@warwick.ac.uk

M. O. Weickert

Division of Metabolic \& Vascular Health, Warwick Medical School, University of Warwick, Coventry, UK

\section{Introduction}

One in three Americans born after 2000 and some $50 \%$ of high-risk ethnic populations are expected to develop type 2 diabetes (T2DM) [1]. Insulin resistance (IR) plays a major role in the development of T2DM, appearing 10-20 years before the onset of the disease [2-4]. IR is mainly caused by adiposity from chronic and excessive energy intake, in the absence of adequate physical activity. Consequent weight gain and excess intra-abdominal fat strongly predispose to IR [5] and finally the development of T2DM [6•, 7].

Examples for strong diet-related modulators of IR are relevant and sustained weight loss; and strenuous exercise with insulin sensitizing effects for up to 72-h $[8,9]$. Large effects on IR might be detected by various methods, which include simple and relatively crude estimates of IR such as simple indices containing fasting glucose and insulin levels. However, for the detection of more subtle changes in IR, gold standard methods such as euglycemic hyperinsulinemic clamps (EHC) and stable isotopes appear to be more sensitive $[10 \bullet, 11 \bullet \bullet]$. In this paper a brief overview of commonly used methods for the assessment of IR in the context of lifestyle and nutrition research in humans is given, with discussion of practicability, suitability and sensitivity of these methods in dietary interventions and epidemiological studies.

Definition of IR and Considerations when using Estimates of IR

Insulin sensitivity is the body's ability to react dynamically to changes in insulin, which is a pre-requisite for maintenance of healthy glucose levels. An individual that requires small changes in insulin to reduce elevated levels of plasma glucose is referred to as insulin sensitive. Conversely, subjects who require large amounts of insulin to affect a small change of in vivo glucose levels are insulin resistant. Those that are highly insulin resistant will either require a large pancreatic response or interventions to improve insulin action. 
Insulin increases glucose use in skeletal muscle and adipose tissue; therefore, a direct measurement of whole-body IR is the euglycemic hyperinsulinemic clamp [12] where glucose infusion rates (GIR) under conditions of defined insulin infusion reflect the ability of the skeletal and adipose tissues' uptake of the infused amount of glucose. By reducing gluconeogenesis (GNG; glucose from non-carbohydrates) and glycogenolysis in the liver, insulin also regulates hepatic or endogenous glucose production (EGP), with renal contributions in humans being small [13].

The ability of insulin to promote glucose clearance is a concentration-rate limited process; the dynamics of this highly responsive, non-linear saturation based mechanism must be kept in mind when a study is considering which of the many methods of measuring IR it is to use. This is particularly important to nutritional interventions, given the limited strength of the stimuli and the duration over which they may act [14•]. A lack of sensitivity in the measurement method may miss a true response amongst the natural variation and measurement noise.

\section{Investigation of Diet-induced Changes in IR}

Sustained changes in body weight and abdominal fat mass are known to be strong modulators of IR. However, even in the general population sustained weight loss with any dietary strategy is difficult to achieve, and additional problems in patients with obesity and T2DM have been recognized [15-19]. Furthermore, many obese patients with T2DM are typically sedentary and may have relevant barriers to exercising $[15,20]$. Therefore, the typical weight loss trial in patients with T2DM either shows yo-yoing with an initially successful weight loss followed by a plateau after 4-6 months and subsequent weight regain [15], or no relevant weight loss at all [21].

Given these difficulties, people who aim to improve IR and their risk of developing T2DM - by lifestyle measures, chose or are advised to use methods that can be more realistically achieved. These include the participation in regular structured exercise programs or changes in the macronutrient composition and quality of foods that have been shown to modulate IR, independent of weight loss [9, 11••, 22-25]. These interventions appear to result in more moderate but relevant changes in IR, as indicated by the results from prospective cohort studies. As an example, intake of diets high in cereal fiber is associated with a significant $30 \%$ reduction of the risk of developing T2DM [26, 27], whereas high protein diets appear to increase this risk by $30 \%$ [28•], despite their known beneficial effects on satiety and short term weight loss $[11 \bullet \bullet]$.

Research on modulators of IR by diet and life style changes is rapidly growing, and also attracting wide coverage by the lay press. However, many of the recommendations are derived from animal models or in vitro studies, or epidemiological studies that do not allow causality to be established [20]. Measuring the often relatively modest effects of weight loss, independent of diet-induced changes in IR, can be challenging given that commonly used surrogate markers of IR may not be sensitive enough to detect differences between dietary groups, whilst significant effects are detected using gold standard methods (e.g., euglycemic hyperinsulinemic clamps (EHC) and stable isotope methods) in the same setting $[10 \bullet \bullet, 11 \bullet \cdot$. However, these methods are time intense and require specific expertise which is not commonly available. Furthermore, considerable costs are involved, with funding in nonpharmacological nutritional interventions often being limited. Therefore, many dietary interventions report results from simple to obtain surrogates of IR only or, when using gold standard methods, the number of participants is typically small.

\section{Gold Standard Measurements IR}

Two basic methods are available to directly assess insulin sensitivity/resistance in nutritional studies, namely: glucose clamp methods for the assessment of whole-body IR, covering skeletal muscle and adipose tissue; and isotope methods for hepatic IR.

\section{Whole-body IR}

The gold-standard for insulin sensitivity is the Euglycemic Hyperinsulinemic Clamp (EHC) [12]. It was developed in response to the inability of markers of IR to account for the highly dynamic relationship between insulin and glucose (see Section G/I Ratio below). The EHC method attempts to control plasma glucose levels under the influence of elevated insulin plasma levels, with glucose infusion rates (GIR) under steady-state conditions defining IR expressed as M-value. A cut-off for defining IR used in can be derived from the literature, but is better defined from M-values in a large cohort of subjects with varying metabolic states.

A criticism of EHC is that the method is impractical and requires relevant resources beyond that for large studies. This is best viewed through description of a standard clamp experiment. EHCs are performed after an overnight fast and avoidance of relevant exercise for 72-hours preceding the clamp. Given the known second meal effects of various foods on insulin sensitivity [29-35], standardization of the evening meal reduces measurement noise. Two intravenous catheters are inserted contra-laterally, and a priming bolus insulin infusion, related to the body surface area of the participant, is given at -10-min. Insulin levels are then maintained through constant infusion until steady-state conditions are achieved. This period can vary depending on subject response and 
experimental control but is typically aimed to be achieved from 120-min. The infusion rate can be varied, with higher insulin infusion rates covering muscle and fat activity, whereas low doses will include a hepatic response. In non-diabetic subjects, EHCs are commonly performed at an infusion rate of $40 \mathrm{mU} \mathrm{kg}^{-1} \mathrm{~min}^{-1}$. Throughout the experiment blood samples are drawn and, capillary glucose measurements are being used for adjusting GIR. The M-value (mean GIR during steady state) is estimated during the final period of the clamp.

Whilst technical complexity and cost are the most cited reasons for the need for an alternative measurement method, it is not the only drawback. The clamp method is highly dependent upon emotional, dietary and physical history; therefore, it is important that subjects are fasted, without activity and lack of distraction from emotional influences (e.g., exciting movies, mobile phones etc.). The equipment required is nontrivial and the environment may be perceived as discomforting by the participant. Therefore, subject adoption and compliance need to be considered, with particular consequences on drop-out rates in cross-over experiments. The risk of a subject becoming hypo- or hyperglycemic must be considered. In prolonged clamps IR typically changes, with the need to keep clamp duration comparable in cross-over studies; however, this can be challenging since reaching steady state conditions varies, even within subjects, and is not predictable. If this is not achieved, measurements cannot be used to compare subjects or groups. Finally, in the hyperinsulinemic clamp the beta cell function is over-ridden, with the need of alternative methods when investigating dynamic aspects of the insulin response [36, 37]. It should be noted that standard EHC only maintains a single insulin concentration, when there are in fact a range of insulin glucose responses. A stepped infusion achieving steady-state at specific increment would be required, which is challenging to achieve [37, 38].

Furthermore, to yield reliable and reproducible results, the potential intake of drugs that influence IR, phase of the menstrual cycle in fertile women, and time of the day when conducting the clamp need to be considered. Finally, the least invasive method for series blood sampling during prolonged clamps is important, which may require use of hand warmers and retrograde catheters to obtain arterialized blood. Automated control of the infusion in response to real-time measurement has been suggested [39-43]; however, these systems have not progressed beyond research projects [43].

Whilst the procedure of a simple EHC is relatively straight forward, more sophisticated setups are sometimes needed, e.g., in an attempt to exclude the confounding effects of physiological secretion of insulin and other hormones to a nutritional stimulus. Nutrient induced insulin secretion interferes with the measurement of EGP and particularly of GL, which is sensitive even to very small increases in insulin [44-46]. Blocking endogenous insulin secretion by infusion of somatostatin, and replacement of hormones such as insulin, glucagon and growth hormone in defined concentrations can be used to detect effects of the respective nutrients on liver IR [44, 47-49].

Figure 1 shows an example of the setting in a somatostatininsulin-glucagon clamp with additional use of both orally and intravenously administered stable isotopes for the measurement of liver IR (EGP and contribution of GNG to EGP, with glycogenolysis being calculated).

\section{Hepatic IR}

The gold standard method for measuring hepatic IR (HEP-IR) includes tracer dilution studies incorporating stepped clamps, which is not feasible in larger metabolic studies and would definitely not be suitable for routine clinical use [36]. Estimated HEP-IR, defined as the product from fasting plasma insulin and EGP [50], has been suggested [51, 52], but is still time consuming and expensive, requiring highly skilled staff with specialized equipment [53••].

To measure EGP, and its components gluconeogenesis (GNG) and glycogenolysis (GL), combined tracer methods are needed. It is a long process typically lasting from 5-hours, plus a $\sim 2$-hours stabilization period. In the example in Fig. 1 the subject was given a weight adjusted priming dose of the glucose isotope ([6,6-2H2]glucose), followed by continuous infusion of the tracer until the end of the measurement. At $-150 \mathrm{~min}$, a second isotope, $\left({ }^{2} \mathrm{H}_{2} \mathrm{O}\right)$ was ingested, adjusted to the body water content and divided into four equal doses spaced at intervals of $45 \mathrm{~min}$ [54], for the assessment of GNG. Precautions must be taken throughout the study to maintain isotopic steady state [55]. Enrichments of ${ }^{2} \mathrm{H}$ in the hydrogens bound to carbon 2 (C2) and carbon 5 (C5) of blood

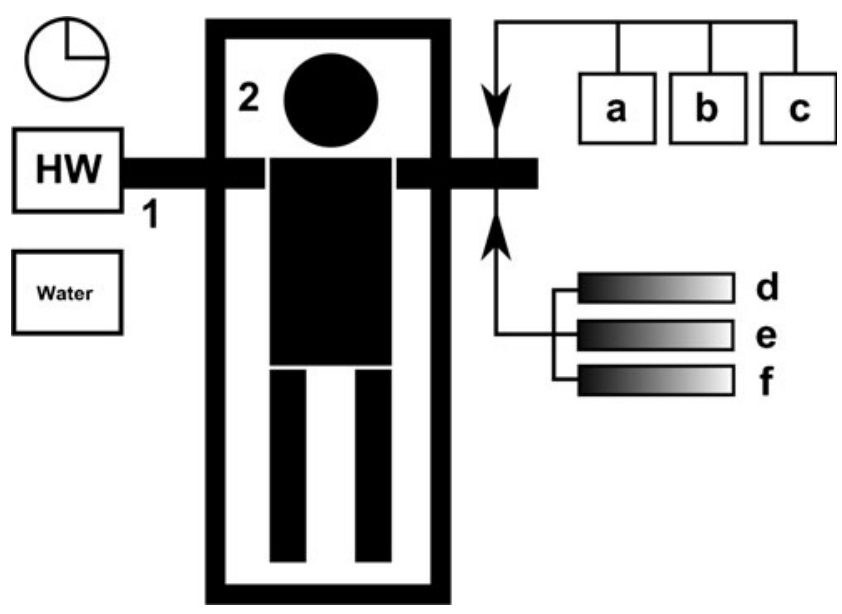

Fig. 1 Schematic of subject undergoing a somatostatin-insulin-glucagon clamp with two different stable isotopes; HW - hand warmer; water - 2H20 enriched water; stop-watch symbol; 1 and 2 are blood sample points (venous blood and capillary samples from hyperemic ear lobe); a-c volumetric infusion pumps ( $\mathrm{a}$ - enriched glucose, $\mathrm{b}-$ spare, $\mathrm{c}$ - intervention infuser); $\mathrm{d}-\mathrm{f}$, infusion syringes ( $\mathrm{d}$ - insulin, e and $\mathrm{f}$ - hormone replacements) 
glucose can then be measured as previously detailed $[55,56]$. Further details need to be considered [44], but describing these is beyond the scope of this review. If steady-state conditions are achieved, EGP can be calculated from the tracer infusion rate of D- $\left[6,6{ }^{2} \mathrm{H}_{2}\right]$ glucose and its enrichment to the hydrogens bound to carbon 6 divided by the mean percent enrichment of plasma D- $\left[6,6{ }^{2} \mathrm{H}_{2}\right]$ glucose. However, a prerequisite for using these simple equations is a constant tracer-to-tracee ratio at the end of the experiment. Otherwise, more complex non-steady state equations need to be used, with ongoing controversies in some of the details [57-60].

The percent contribution of GL to EGP can then be set to the ratio of ${ }^{2} \mathrm{H}$ enrichment at $\mathrm{C} 5$ to that at $\mathrm{C} 2$, and the percent contribution of GL is calculated as $[1-(\mathrm{C} 5 / \mathrm{C} 2)] \times 100$ [44], whereas absolute contributions of GNG and GL are calculated by multiplying their percent contributions by the rate of EGP [61]. Another commonly used parameter is the metabolic clearance rate of glucose (expressed in $\mathrm{ml} \cdot \mathrm{kg}^{-1} \cdot \mathrm{min}^{-1}$ ), which can be calculated from the rate of disappearance of glucose divided by the mean glucose concentration over the respective time period [62]. Combination of stable isotope methods with EHC allows insulin suppression of EGP and its components to be measured.

\section{Simple Surrogate Indices of IR}

\section{Indirect IR Indices Under Fasted Conditions}

Most commonly used indices of IR are based purely upon fasting concentration of insulin, glucose or both measured in plasma; with data being manipulated through simple equations to yield an IR indicator. These indices include the inverse of fasting plasma insulin [63] (i.e., 1/FPI), or the fasting glucose-insulin (G/I) ratio, i.e., FPG/FPI, [64]. Under normal physiological conditions, fasting plasma glucose exhibits limited variation. Conceptually, such simple indices appear logically sound, with fasting insulin levels expected to rise in subjects becoming insulin resistant. However, sensitivity of these indices in comparison to gold standard methods is poor $[10 \bullet, 11 \bullet \cdot]$ and there are additional flaws when used in diabetic subjects [38].

\section{Homeostasis Model Assessment}

The most adopted method for the estimation of IR is arguably the homeostasis model assessment (HOMA). It was introduced in 1985 [65], using a computer program incorporating several sites of insulin and glucose dynamics (glucose uptake in brain, glucose and insulin uptake in fat and muscle; and interaction between liver and pancreatic beta-cells). The response functions for each of these organs (or sub-systems) are derived from population norms. This full model is highly nonlinear, therefore, through a series of steady-state approximations under fasted conditions the authors derived a simplified system, referred to as HOMA-1, consisting of two linear equations giving insulin resistance (HOMA-IR) and beta-cell function (HOMA-B). The equations are:

$\mathrm{HOMA}-\mathrm{R}=\mathrm{FPI} \times \mathrm{FPG} / \alpha$,

HOMA-B $=(\beta \times$ FPI $) / \gamma$

where $\alpha, \beta$ and $\gamma$ are normalization constants, dependent on measurement units that ensure the value for HOMA-IR being normalized between 1 and $100 \%$ for a "normal" individual. An excellent review of the issues involving the use and 'abuse' of the method is provided by the original research team [66]. To summarize, whilst HOMA has been shown to be successful in detecting changes in IR and beta-cell function in independent cohorts [67], individual longitudinal adaption [68] and single ethnicity epidemiological studies [69], difficulties arise when considering cohorts of mixed ethnicity or comparing differences in ethnic groups, as the normality defined for HOMA may not hold for sub-groups; or application in small samples. In addition, due to influences on the underlying dynamics when subjects are beta-cell impaired, or treated with insulin or secretagogues, care must be taken when interpreting the HOMA output.

Further indices have been derived from the HOMA original model; HOMA2 [70] is the full implementation of the original HOMA model. Unlike the more succinct HOMA equations which can be calculated by hand, HOMA2 requires specific software written by the authors, which can be downloaded from the Diabetes Trials Unit, University of Oxford. Cpeptide HOMA [71] has also been proposed, in an effort to avoid confounding factors of hepatic clearance [68, 72].

An alternative model for insulin sensitivity was present in 1999 called Quantitative Insulin Sensitivity Check Index (QUICKI - [73]), derived from correlation and analysis of FSIVGTT data which revealed a skewed relationship between fasting plasma and glucose; the authors developed the following equations:

QUICKI $=1 /[\log ($ FPI $)+\log ($ FPG $)]$.

If the reciprocal of the HOMA-IR model is given as insulin sensitivity and the natural log taken, through simple manipulation it can be seen that these two equations are mathematical identical under constant of proportionality. Therefore, this model adds no further information above HOMA-IR [66].

\section{Quasi-Dynamic Indices}

A series of indices which attempt to capture the dynamic relationship, as opposed to enforcing steady-state conditions 
through fasted plasma sampling, have been suggested [50, 74-76]. The oral glucose tolerance test (OGTT) method is predominantly used, due to subject acceptance and simplicity of the protocol. Rather than adopting series analyses of the insulin-glucose dynamics, these indices attempt to capture the underlying interplay between the hepatic and peripheral systems, by creating simple point estimates from the samples (e.g., means and area under the curve). Comparative studies investigating these types of markers [50] have indicated that they offer an improvement on the steady-state equivalents (e.g., HOMA, G/I ratio) when compared to the clamp methods discussed previously. However, the natural variation and noise that is introduced through the oral ingestion method (e.g., absorption rates, gastric emptying, and glucose uptake) must be considered.

\section{Matsuda Index}

An index which attempts to incorporate both hepatic and muscle insulin-glucose interactions under the OGTT impulse was present in 1999 [74]. The hepatic response is represented by the fasted plasma and glucose concentrations prior to the OGTT, with the muscle response represented through the mean values of plasma and insulin over the

$\operatorname{ISI}($ Matsuda $)=10000 / \operatorname{sqrt}\left(I_{0} \times \mathrm{G}_{0} \times \mathrm{I}_{\mathrm{m}} \times \mathrm{G}_{\mathrm{m}}\right)$

where I represents insulin, G plasma glucose; subscript 0 denotes the fasting value, i.e., at time point 0 ; subscript $\mathrm{m}$ is the mean taken over the OGTT time course, generally 120 $\min$. The numerator attempts to keep the index in a comparable range to $\mathrm{EHC}$.

\section{Supplemented Indices}

Several simple indices have been suggested that supplement the OGGT measurements with common physiological measurement, to improve the applicability of the indices. The Stumvoll Index [75], defined as,

$\mathrm{MCR}_{\text {est }}=18.8-0.27 \times \mathrm{BMI}-0.0052 \mathrm{I}_{120}-0.27 \times \mathrm{G}_{90}$

introduces variation in body size, through BMI, to insulin measure at the 120-min sample and glucose at 90-min to produce a combined.

A complex variation on this was suggested by [76].

$\mathrm{ISI}=\left[\mathrm{A}+\mathrm{B} \times \mathrm{BW} \times F P I-\mathrm{C} \times \mathrm{BW} \times \mathrm{AUC}_{\mathrm{g}}-\mathrm{U}_{\mathrm{g}}\right] /\left[\mathrm{AUC}_{\mathrm{i}} \times \mathrm{BW}\right]$

if standard units are used ( $\mathrm{kg}, \mathrm{mmol}$, liter and hours), $\mathrm{A}=520$, $\mathrm{B}=1.9 / 6$ and $\mathrm{C}=1.9 / 18$. This equation requires body weight
(BW), glucose and insulin area under the curve $\left(\mathrm{AUC}_{\mathrm{g}}, \mathrm{AUC}_{\mathrm{i}}\right.$, and $\mathrm{U}_{\mathrm{g}}$ ) from an OGGT.

Hepatic Insulin Resistance

Surrogate indices for HEP-IR have been presented due to the increased difficulties of isotope manipulation. Vangipurapu, Stančáková [52] suggest an index of HEP-IR generated through stepwise regression, which moderately correlates with EGP x FPI. Abdul-Ghani, Matsuda [77] propose the use of the Matsuda Index [50] as an indicator, not predictor, of HEP-IR. The use of correlation analyses to validate these indices does not yield predictive power, reliability or robustness, beyond the cohort they are developed against. When validated independently these indices perform poorly; indeed, simple plasma measurements or HOMA-IR can be used to achieve improved co-efficient of determination, mean squared error and co-efficient of variation [53••].

\section{Mathematical Models of IR}

The indices described so far generally rely on, or assume, steady-state conditions; to investigate the dynamic response of subjects to glucose challenges alternative methods of mathematical analyses are required. The most common method is compartmental modeling - where the material is assumed to move instantaneously, through homogeneously mixed pools, or compartments [78]. A model of this type has already been discussed, namely, the HOMA model; HOMA-IR and HOMA-B formulae are derived from a more complete dynamic mathematical model, referred to as HOMA2.

\section{Oral Glucose Insulin Sensitivity (OGIS)}

The OGIS model [79] describes the glucose kinetics during an OGGT ( 2 or $3 \mathrm{hr}$ ); the model consists of a single compartment representing the plasma glucose pool,

$\mathrm{VG}(\mathrm{t})^{\prime}=-C l(\mathrm{t}) \mathrm{G}(\mathrm{t})+\mathrm{R}_{\mathrm{a}}(\mathrm{t})$

where ' represents first derivative, $\mathrm{t}$ time, $\mathrm{V}$ the volume of distribution, $\mathrm{G}(\mathrm{t})$ the glucose concentration, $\mathrm{Cl}(\mathrm{t})$ the clearance rate at time (t) and $\mathrm{R}_{\mathrm{a}}(\mathrm{t})$ the rate of appearance of glucose. The rate of clearance $(\mathrm{Cl})$ is then assumed to have an affine relationship with changes in plasma insulin changes, i.e., $\mathrm{Cl}=\mathrm{Cl}_{\mathrm{b}}+\mathrm{S} \Delta \mathrm{I}$, where $\mathrm{Cl}_{\mathrm{b}}$ is basal glucose clearance, $\Delta \mathrm{I}$ the change in insulin and $\mathrm{S}$ is simple the gradient between $\mathrm{Cl}$ and $\Delta \mathrm{I}$. These equations are then combined to derive an equation for glucose clearance that can be considered as a surrogate index. Due to its simplicity (three blood samples required), it can be a useful index for IR in large epidemiological settings; however, the model requires 
parameters that cannot be estimated, they must be assumed, raising questions over natural population variation.

\section{Minimal Model}

The 'Minimal Model' was introduced in the 1980s by Bergman and Cobelli $[80,81]$. It is widely used but has practical issues if there is insufficient interaction between insulin and glucose, e.g., in T2DM or obese subjects [82]. The most common form for these equations is:

$$
\begin{array}{ll}
\mathrm{G}(\mathrm{t})^{`}=\mathrm{X}(\mathrm{t}) \times \mathrm{G}(\mathrm{t})+\mathrm{p}_{1} \times\left(\mathrm{G}_{\mathrm{b}}-\mathrm{G}(\mathrm{t})\right) & \\
\mathrm{X}(\mathrm{t})^{\prime}=\mathrm{p}_{3} \times\left(\mathrm{I}(\mathrm{t})-\mathrm{I}_{\mathrm{b}}\right)-\mathrm{p}_{2} \times \mathrm{X}(\mathrm{t}) & \\
\mathrm{I}(\mathrm{t})^{\prime}=\gamma \times(\mathrm{G}(\mathrm{t})-\mathrm{h}) \times \mathrm{t}-\mathrm{n} \times \mathrm{I}(\mathrm{t}) & \text { if } \mathrm{G}(\mathrm{t})>=\mathrm{h} \\
\mathrm{I}(\mathrm{t})^{`}=\mathrm{n} \times \mathrm{I}(\mathrm{t}) & \text { if } \mathrm{G}(\mathrm{t})<\mathrm{h}
\end{array}
$$

where $t$ is time, $G(t)$ is the plasma glucose at time $t, I(t)$ the plasma insulin production that has two forms depending on glucose concentration; $\mathrm{X}(\mathrm{t})$ is a remote compartment, that is unspecified in biological terms; $b$ denotes basal levels; $n$ is the insulin clearance fraction, $\gamma$ is a indication of pancreatic response to glucose and $\mathrm{h}$ the basal glucose plasma concentrations. Parameters $\mathrm{p}_{1}, \mathrm{p}_{2}$ and $\mathrm{p}_{3}$ and the initial glucose concentration can be estimated from FSIVGTT data; this may be performed in any suitable software package. Insulin sensitivity is defined as the ratio of parameters $\mathrm{p}_{3} / \mathrm{p}_{2}$. A tool for the calculation is provided, MINMOD [83].

\section{Conclusions}

Whilst simple surrogate indices meet the pre-requisite of being low cost and relatively simple to perform, in comparison to $\mathrm{EHC}$ and tracer methods, they do not provide the required level of sensitivity and accuracy, given the relatively subtle variation dietary interventions offer. In addition, they are not able to reliably differentiate between whole-body and HEP-IR. Dynamic mathematical models offer an interesting perspective, particularly when considering the prandial phase. However, difficulties arise with the intensive sampling procedures and complex analyses - making the transition to the full clamp technique feasible. Recent advances in mathematical analysis which develop models encapsulating organ level dynamics may prove beneficial as they offer dynamic interactions at a more insightful granularity (e.g., muscle, liver, brain, etc.); these models are called physiological based models [86].

\section{Compliance with Ethics Guidelines}

Conflict of Interest John G. Hattersley and Martin O. Weickert declare that they have no conflict of interest.
Human and Animal Rights and Informed Consent This article does not contain any studies with human or animal subjects performed by any of the authors.

\section{References}

Papers of particular interest, published recently, have been highlighted as:

- Of importance,

•. Of major importance

1. Narayan KV, Boyle JP, Thompson TJ, et al. Lifetime risk for diabetes mellitus in the United States. JAMA. 2003;290(14):1884-90.

2. Shulman GI. Cellular mechanisms of insulin resistance. J ClinInvest. 2000;106(2):171-6.

3. Warram JH, Martin BC, Krolewski AS, et al. Slow glucose removal rate and hyperinsulinemia precede the development of type II diabetes in the offspring of diabetic parents. Ann Intern Med. 1990;113(12): 909-15.

4. Lillioja S, Mott DM, Howard BV, et al. Impaired glucose tolerance as a disorder of insulin action. N Engl J Med. 1988;318(19):1217-25.

5. McAuley K, Mann J. Thematic review series: patient-oriented research. Nutritional determinants of insulin resistance. J Lipid Res. 2006;47(8):1668-76.

6. - Sung K-C, Jeong W-S, Wild SH, et al. Combined influence of insulin resistance, overweight/obesity, and fatty liver as risk factors for type 2 diabetes. Diabetes Care. 2012;35(4):717-22. Authors show that clustering of IR, overweight/obesity and fatty liver markedly increases the risk of developing T2DM, with independent effects of the single components.

7. Vazquez G, Duval S, Jacobs DR, et al. Comparison of body mass index, waist circumference, and waist/hip ratio in predicting incident diabetes: a meta-analysis. Epidemiol Rev. 2007;29(1):115-28.

8. Moore MC, Cherrington AD, Wasserman DH. Regulation of hepatic and peripheral glucose disposal. Best Pract Res Clin Endocrinol Metab. 2003;17(3):343-64.

9. Sivaraman S, Weickert MO. Nutrition and exercise in the treatment of type 2 diabetes mellitus. Hamdan Med J. 2012;5(2):131-44.

10. • Johnston K, Thomas E, Bell J, et al. Resistant starch improves insulin sensitivity in metabolic syndrome. Diabet Med. 2010;27(4): 391-7. Authors show that treatment with resistant starch for 12-wk significantly increases whole-body IR by $15 \%$, as mesured using EHC. This effect would have been missed using markers of IR only, including HOMA $\%$ S and HOMA $\%$ B.

11. •• Weickert MO, Roden M, Isken F, et al. Effects of supplemented isoenergetic diets differing in cereal fiber and protein content on insulin sensitivity in overweight humans. Am J Clin Nutr. 2011;94(2):459-71. We show that treatment with isoenergetic diets varying in cereal fiber and protein content for 6 to 18-wk results in significantly and markedly changed whole-body IR (25\% difference between groups in $M$-value), and smaller but significant differences in EGP. These effects would have been missed using markers of IR only, including HOMA-IR and OGIS.

12. DeFronzo RA, Tobin JD, Andres R. Glucose clamp technique: a method for quantifying insulin secretion and resistance. Am J Physiol. 1979;237(3):E214-23.

13. Perseghin G, Lattuada G, Danna M, et al. Insulin resistance, intramyocellular lipid content, and plasma adiponectin in patients with type 1 diabetes. Am J Physiol Endocrinol Metab. 2003;285(6): E1174-81. 
14. - Weickert MO. What dietary modification best improves insulin sensitivity and why? Clin Endocrinol. 2012;77(4):508-12. A concise overview of effects of diet on IR.

15. Pi-Sunyer X, Blackburn G, Brancati F, et al. Reduction in weight and cardiovascular disease risk factors in individuals with type 2 diabetes: one-year results of the look AHEAD trial. Diabetes Care. 2007;30(6): 1374-83.

16. Heshka S, Yang M-U, Wang J, et al. Weight loss and change in resting metabolic rate. Am J Clin Nutr. 1990;52(6):981-6.

17. Bray G. Effect of caloric restriction on energy expenditure in obese patients. Lancet. 1969;294(7617):397-8.

18. Nair K, Halliday D, Garrow J. Increased energy expenditure in poorly controlled type 1 (insulin-dependent) diabetic patients. Diabetologia. 1984;27(1):13-6.

19. Bogardus C, Taskinen M-R, Zawadzki J, et al. Increased resting metabolic rates in obese subjects with non-insulin-dependent diabetes mellitus and the effect of sulfonylurea therapy. Diabetes. 1986;35(1):1-5.

20. Weickert MO. Nutritional modulation of insulin resistance. Scientifica. 2012. doi:10.6064/2012/424780.

21. Milne RM, Mann JI, Chisholm AW, et al. Long-term comparison of three dietary prescriptions in the treatment of NIDDM. Diabetes Care. 1994;17(1):74-80.

22. Kastorini C-M, Milionis HJ, Esposito K, et al. The effect of mediterranean diet on metabolic syndrome and its componentsa metaanalysis of 50 studies and 534,906 individuals. J Am Coll Cardiol. 2011;57(11):1299-313.

23. Thomas HE, Kay TW. Intracellular pathways of pancreatic $\beta$ - cell apoptosis in type 1 diabetes. Diabetes/Metabol Res Rev. 2011;27(8): 790-6.

24. Risérus U, Willett WC, Hu FB. Dietary fats and prevention of type 2 diabetes. Prog Lipid Research. 2009;48(1):44-51.

25. Weickert MO, Pfeiffer AF. Metabolic effects of dietary fiber consumption and prevention of diabetes. J Nutr. 2008;138(3): 439-42.

26. de Munter JS, Hu FB, Spiegelman D, et al. Whole grain, bran, and germ intake and risk of type 2 diabetes: a prospective cohort study and systematic review. PLoS Med. 2007;4(8):e261.

27. Schulze MB, Schulz M, Heidemann C, et al. Fiber and magnesium intake and incidence of type 2 diabetes: a prospective study and metaanalysis. Arch Intern Med. 2007;167(9):956.

28. - Sluijs I, Beulens JW, Spijkerman AM, et al. Dietary intake of total, animal, and vegetable protein and risk of type 2 diabetes in the European Prospective Investigation into Cancer and Nutrition (EPIC)-NL study. Diabetes Care. 2010;33(1):43-8. Prospective cohort study in 38,094 participants, suggesting 30\% increased Diabetes risk with diets high in animal protein but also in total protein.

29. Robertson M, Currie J, Morgan L, et al. Prior short-term consumption of resistant starch enhances postprandial insulin sensitivity in healthy subjects. Diabetologia. 2003;46(5):659-65.

30. Weickert M, Mohlig M, Koebnick $C$, et al. Impact of cereal fibre on glucose-regulating factors. Diabetologia. 2005;48(11): 2343-53.

31. Freeland KR, Anderson GH, Wolever T. Acute effects of dietary fibre and glycaemic carbohydrate on appetite and food intake in healthy males. Appetite. 2009;52(1):58-64.

32. Hamedani A, Akhavan T, Samra RA, et al. Reduced energy intake at breakfast is not compensated for at lunch if a high-insoluble-fiber cereal replaces a low-fiber cereal. Am J Clinical Nutr. 2009;89(5): 1343-9.

33. Arai H, Mizuno A, Sakuma M, et al. Effects of a palatinose-based liquid diet (Inslow) on glycemic control and the second-meal effect in healthy men. Metabolism. 2007;56(1):115-21.

34. Brighenti F, Benini L, Del Rio D, et al. Colonic fermentation of indigestible carbohydrates contributes to the second-meal effect. Am J Clinical Nutr. 2006;83(4):817-22.
35. Östman EM, Elmståhl HGL, Björck IM. Barley bread containing lactic acid improves glucose tolerance at a subsequent meal in healthy men and women. J Nutr. 2002;132(6):1173-5.

36. Pacini G, Mari A. Methods for clinical assessment of insulin sensitivity and beta-cell function. Best Pract Res Clin Endocrinol Metab. 2003;17(3):305-22.

37. Ahren B, Pacini G. Importance of quantifying insulin secretion in relation to insulin sensitivity to accurately assess beta cell function in clinical studies. Eur J Endocrinol. 2004;150(2):97-104.

38. Muniyappa R, Lee S, Chen $\mathrm{H}$, et al. Current approaches for assessing insulin sensitivity and resistance in vivo: advantages, limitations, and appropriate usage. Am J Physiol Endocrinol Metab. 2008;294(1): E15-26.

39. Bequette BW. Glucose clamp algorithms and insulin time-action profiles. J Diabetes Sci Technol. 2009;3(5):1005-13.

40. Furler S, Zelenka G, Kraegen E. Development and testing of a simple algorithm for a glucose clamp. Med Biol Eng Comput. 1986;24(4): 365-70.

41. Clemens AH, Hough DL, D'Orazio PA. Development of the Biostator Glucose clamping algorithm. Clin Chem. 1982;28(9): 1899-904.

42. Catalogna M, Cohen E, Fishman S, et al. Artificial neural networks based controller for glucose monitoring during clamp test. PLoS One. 2012;7(8):e44587.

43. Sternberg F, Meyerhoff C, Mennel F, et al. Does fall in tissue glucose precede fall in blood glucose? Diabetologia. 1996;39(5):609-12.

44. Weickert MO, Loeffelholz C, Roden M, et al. A Thr94Ala mutation in human liver fatty acid-binding protein contributes to reduced hepatic glycogenolysis and blunted elevation of plasma glucose levels in lipid-exposed subjects. Am J Physiol Endocrinol Metab. 2007;293(4):E1078-84.

45. Cherrington A, Edgerton D, Sindelar D. The direct and indirect effects of insulin on hepatic glucose production in vivo. Diabetologia. 1998;41(9):987-96.

46. Edgerton DS, Cardin S, Emshwiller M, et al. Small increases in insulin inhibit hepatic glucose production solely caused by an effect on glycogen metabolism. Diabetes. 2001;50(8):1872-82.

47. Williamson JR, Browning ET, Scholz R, et al. Inhibition of fatty acid stimulation of gluconeogenesis by $(+)$-decanoylcarnitine in perfused rat liver. Diabetes. 1968;17(4):194-208.

48. Roden M, Stingl H, Chandramouli V, et al. Effects of free fatty acid elevation on postabsorptive endogenous glucose production and gluconeogenesis in humans. Diabetes. 2000;49(5):701-7.

49. Boden G. Role of fatty acids in the pathogenesis of insulin resistance and NIDDM. Diabetes. 1997;46(1):3-10.

50. Matsuda M, DeFronzo RA. Insulin sensitivity indices obtained from oral glucose tolerance testing: comparison with the euglycemic insulin clamp. Diabetes Care. 1999;22(9):1462-70.

51. Miyazaki Y, Glass L, Triplitt C, et al. Abdominal fat distribution and peripheral and hepatic insulin resistance in type 2 diabetes mellitus. Am J Physiol Endocrinol Metab. 2002;283(6):E1135-43.

52. Vangipurapu J, Stančáková A, Kuulasmaa T, et al. A novel surrogate index for hepatic insulin resistance. Diabetologia. 2011;54(3):540-3.

53. • Hattersley JG, Möhlig M, Roden M, et al. Quantifying the improvement of surrogate indices of hepatic insulin resistance using complex measurement techniques. PLoS One. 2012;7(6):e39029. We reviewed $H E P-I R$ indices proposed by others and validated them using an independent cohort, using robust methodologies and statistical analyses; in addition to suggesting improved alternative HEP-IR indices.

54. Hother-Nielsen O, Beck-Nielsen H. On the determination of basal glucose production rate in patients with type 2 (non-insulin-dependent) diabetes mellitus using primed-continuous 3-3H-glucose infusion. Diabetologia. 1990;33(10):603-10.

55. Landau BR, Wahren J, Chandramouli V, et al. Contributions of gluconeogenesis to glucose production in the fasted state. J Clin Invest. 1996;98(2):378. 
56. Chandramouli V, Ekberg K, Schumann WC, et al. Quantifying gluconeogenesis during fasting. Am J Physiol Endocrinol Metab. 1997;273(6):E1209-15.

57. Steele R. Influences of glucose loading and of injected insulin on hepatic glucose output*. Ann N Y Acad Sci. 1959;82(2):420-30.

58. Basu R, Breda E, Oberg AL, et al. Mechanisms of the age-associated deterioration in glucose tolerance contribution of alterations in insulin secretion, action, and clearance. Diabetes. 2003;52(7):1738-48.

59. Livesey G, Wilson P, Dainty J, et al. Simultaneous time-varying systemic appearance of oral and hepatic glucose in adults monitored with stable isotopes. Am J Physiol Endocrinol Metab. 1998;275(4):E717-28.

60. Gastaldelli A, Coggan A, Wolfe R. Assessment of methods for improving tracer estimation of non-steady-state rate of appearance. J Appl Physiol. 1999;87(5):1813-22.

61. Staehr P, Hother-Nielsen O, Landau BR, et al. Effects of free fatty acids per se on glucose production, gluconeogenesis, and glycogenolysis. Diabetes. 2003;52(2):260-7.

62. Radziuk J, Lickley H. The metabolic clearance of glucose: measurement and meaning. Diabetologia. 1985;28(6):315-22.

63. Hermans M, Levy J, Morris R, et al. Comparison of insulin sensitivity tests across a range of glucose tolerance from normal to diabetes. Diabetologia. 1999;42(6):678-87.

64. Legro RS, Finegood D, Dunaif A. A fasting glucose to insulin ratio is a useful measure of insulin sensitivity in women with polycystic ovary syndrome. J Clin Endocrinol Metab. 1998;83(8):2694-8.

65. Matthews DR, Hosker JP, Rudenski AS, et al. Homeostasis model assessment: insulin resistance and beta-cell function from fasting plasma glucose and insulin concentrations in man. Diabetologia. 1985;28(7): 412-9.

66. Wallace TM, Levy JC, Matthews DR. Use and abuse of HOMA modeling. Diabetes care, 2004. 27(6):1487-1495. 68. Group, U.P.D.S., UK prospective diabetes study 16. Overview of 6 years' therapy of type II diabetes: a progressive disease. Diabetes. 1995;44(11):1249-58.

67. Wallace T, Levy J, Matthews D. An increase in insulin sensitivity and basal beta- cell function in diabetic subjects treated with pioglitazone in a placebo- controlled randomized study. Diabet Med. 2004;21(6): 568-76.

68. Haffner SM, Gonzalez C, Miettinen H, et al. A prospective analysis of the HOMA model: the Mexico City diabetes study. Diabetes Care. 1996;19(10):1138-41.

69. Levy JC, Matthews DR, Hermans MP. Correct homeostasis model assessment (HOMA) evaluation uses the computer program. Diabetes Care. 1998;21(12):2191-2.
70. Li X, Zhou Z, Qi H, et al. Replacement of insulin by fasting Cpeptide in modified homeostasis model assessment to evaluate insulin resistance and islet beta cell function. J Central South Uni. 2004;29(4):419.

71. Cobelli C, Toffolo GM, Dalla C. Man, et al., Assessment of $\beta$-cell function in humans, simultaneously with insulin sensitivity and hepatic extraction, from intravenous and oral glucose tests. Am J Physiol Endocrinol Metab. 2007;293(1):E1-E15.

72. Katz A, Nambi SS, Mather K, et al. Quantitative insulin sensitivity check index: a simple, accurate method for assessing insulin sensitivity in humans. J Clin Endocrinol Metab. 2000;85(7): 2402-10.

73. Mari A, Pacini G, Brazzale A, et al. Comparative evaluation of simple insulin sensitivity methods based on the oral glucose tolerance test. Diabetologia. 2005;48(4):748-51.

74. Stumvoll M, Mitrakou A, Pimenta W, et al. Use of the oral glucose tolerance test to assess insulin release and insulin sensitivity. Diabetes Care. 2000;23(3):295-301.

75. Soonthornpun S, Setasuban W, Thamprasit A, et al. Novel insulin sensitivity index derived from oral glucose tolerance test. J Clin Endocrinol Metab. 2003;88(3):1019-23.

76. Abdul-Ghani MA, Matsuda M, Balas B, et al. Muscle and liver insulin resistance indexes derived from the oral glucose tolerance test. Diabetes Care. 2007;30(1):89-94.

77. Jacquez JA. Compartmental analysis in biology and medicine. Ann Arbor: University of Michigan Press; 1985.

78. Mari A, Pacini G, Murphy E, et al. A model-based method for assessing insulin sensitivity from the oral glucose tolerance test. Diabetes Care. 2001;24(3):539-48.

79. Bergman RN, Ider YZ, Bowden CR, et al. Quantitative estimation of insulin sensitivity. Am J Physiol. 1979;236(6):E667-77.

80. Toffolo G, Bergman RN, Finegood DT, et al. Quantitative estimation of beta cell sensitivity to glucose in the intact organism: a minimal model of insulin kinetics in the dog. Diabetes. 1980;29(12):979-90.

81. De Gaetano A, Arino O. Mathematical modelling of the intravenous glucose tolerance test. J Math Biol. 2000;40(2):136-68.

82. Pacini G, Bergman RN. MINMOD: a computer program to calculate insulin sensitivity and pancreatic responsivity from the frequently sampled intravenous glucose tolerance test. Comput Methods Programs Biomed. 1986;23(2):113-22.

83. Levitt DG. PKQuest: a general physiologically based pharmacokinetic model. Introduction and application to propranolol. BMC. Pharmacol Toxicol. 2002;2(1):5. 